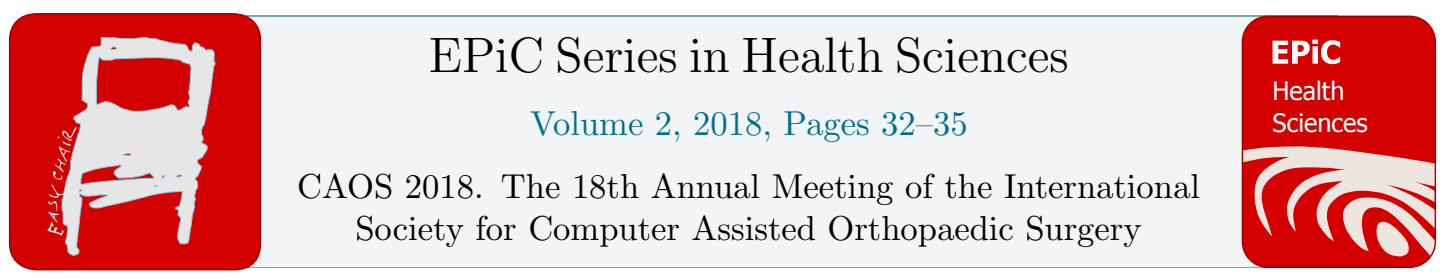

\title{
Learning of A CAOS Enhanced Mechanical Instrument System for Total Knee Arthroplasty: A CUSUM Analysis
}

\author{
Yifei Dai ${ }^{1 *}$, James I. Huddleston, III ${ }^{2}$, Matt Rueff ${ }^{1}$, Laurent Angibaud ${ }^{1}$, \\ Derek F. Amanatullah ${ }^{2 *}$ \\ ${ }^{1 *}$ Exactech Inc, Gainesville, FL 32653, USA \\ ${ }^{2}$ Dept of Orthopaedic Surgery, Stanford University School of Medicine, Redwood City, CA, USA \\ dfa@stanford.edu, yifei.dai@exac.com
}

\begin{abstract}
This study employed an advanced method (Cumulative sum control chart, CUSUM) to analyze the learning curve regarding surgical efficiency (time) in four surgeons from different experience levels upon their adoption of a mechanical instrument enhanced by computer-assisted orthopaedic surgery (CAOS). The first six CAOS-TKA cases of each surgeon were analyzed to identify the case number when their learning process was stabilized (cases to proficiency). The surgical time increase during the learning phase were compared between senior and fellow surgeons. The data demonstrated a short learning duration (2-3 cases) both senior and fellow surgeons and a mild impact from learning compared to cases performed using conventional instrument system.
\end{abstract}

\section{Introduction}

Computer-assisted orthopaedic surgery (CAOS) has been shown to offer increased accuracy and precision to total knee arthroplasty (TKA) compared to the conventional techniques [1]. Despite the promising results, one of the drawbacks for surgeons to adopt CAOS technology may be the requirement of switching from conventional to CAOS-specific instruments. Recent advance in CAOS introduced a system designed to enhance the existing conventional mechanical instruments, removing the need for significant instrument change. While TKA performed by this system can benefit from the improved accuracy offered by CAOS technology, it is important to assess the learning of the system to evaluate the efficiency in its adoption.

To date, limited studies have assessed the learning of new surgical technology. The methodology applied in existing studies usually compared surgical time between the cases performed during learning and those from the later cases, with an assumed duration (number of cases) of the learning curve [2]. 
Researchers also have performed logarithmic regression on the initial CAOS case series to find the duration of the learning phase [3]. However, as the surgical time data often, by nature, noisy, the regression result can be difficult to evaluate.

Cumulative sum control chart (CUSUM) has been widely applied to assess the stabilization of an industrial production process, and proven to be an objective and effective tool to evaluate the learning process. Although many successes have been achieved by this method in other medical fields [4], the analysis is currently under-recognized in TKA research. The purpose of this study was to use CUSUM to assess the learning curve on the critical surgical steps using a new CAOS enhanced mechanical instrument system.

\section{Materials and Methods}

Two senior surgeons and two fellow surgeons with no prior experiences in CAOS TKA were included in this study. Each surgeon performed proximal tibial and distal femoral resections on six knee models (MITA knee insert, Medical Models, Bristol, UK) using conventional mechanical instruments and six knee models under the guidance of a CAOS system (ExactechGPS ${ }^{\circledR}$ TKA Plus, Blue-Ortho, Gieres, FR), which was designed to be used with the same conventional mechanical instrument system. All resections were created targeting $0^{\circ}$ varus/valgus in both tibia and femur, $0^{\circ}$ femoral flexion/extension, $3^{\circ}$ tibial posterior slope, and $10 \mathrm{~mm}$ resection depth.

To perform CUSUM analysis, the 6 CAOS guided cases from each surgeon were ordered chronologically. The cumulative sum of deviances was calculated as following [4]: The CUSUM of the first case was the difference between the surgical of the first case and the mean surgical time of all 6 cases. The CUSUM of the second cases was the previous case's CUSUM plus the difference between the surgical time of the second case and the mean surgical time. This recursive process continued until the last case (\#6), which was calculated as 0. CUSUM value was plotted in chronological order for each surgeon. A horizontal trend in the plot signified the deviances were equally balanced around 0 , indicating the process was operating with stability. The case number (cases to proficiency) by which the CUSUM value entered the horizontal trend was identified as the end of learning curve for each surgeon. The cases to proficiency was compared between the senior surgeons and the fellow surgeons. The surgical time in CAOS guided cases during and after learning was compared to the conventional cases within each surgeon (due to limited cases number per surgeon, statistical assessment of the differences was not performed). The increase in surgical time after learning the CAOS system was compared to conventional cases on the pooled data (significance defined as $\mathrm{p}<0.05$ ).

\section{Results}

The CUSUM plot exhibited three unique phases in the first six cases of each surgeon, with Phase II demonstrating stabilization of the process (Fig 1). No substantial difference between the senior surgeon and novice surgeon groups was found in the speed of mastering the CAOS system (2-3 cases). However, compared to the senior surgeons, the fellow surgeons exhibited slightly steeper learning curve by adding 3-4 minutes more to their learning cases (Fig 1,2). Compared to the conventional TKA, adding CAOS technology to conventional instruments slightly increased surgical time by 4-6 minutes during learning, and the difference reduced to 2-3 minutes after learning. No significant difference in surgical time was found between senior and fellow surgeons after they mastered the CAOS system (Fig 2B). 


\section{Discussion}

This study applied CUSUM method to analyze learning curve of a CAOS enhanced mechanical instrument system for TKA, viewing learning of the system as a process that eventually stabilizes surgical time in critical TKA steps. As the CAOS guidance is based on existing conventional mechanical instruments, the adoption of the technology exhibited minimum learning effort (2-3 case to learn), independent of the surgeon's experience level. Compared to conventional cases performed using the same mechanical instrument system, using the CAOS enhanced system moderately increased the surgical time in critical bony resection steps by 4-6 minutes during learning. After quick mastering of the technology, the surgical time was only slightly extended by 2-3 minutes compared to conventional cases. The results demonstrated minimum extension of operative time by introducing CAOS guidance to the existing conventional mechanical instruments, offering the proven benefit of CAOS technology without major disruption in the surgical tools the surgeons are already familiar with.

The CUSUM method was developed in the 1970s for analyzing learning curve for surgical procedures [5,6], and since then being applied to various medical fields [4], however the use of this method in TKA is very limited [7]. Utilization of this advanced method in studying learning curve can provide improved understanding of CAOS learning in general but also allows differences in learning between individual surgeons to be explored. Further investigation of this study may include expanding the CUSUM assessment to the entire TKA surgical duration with more surgeon groups with different characteristics.

A

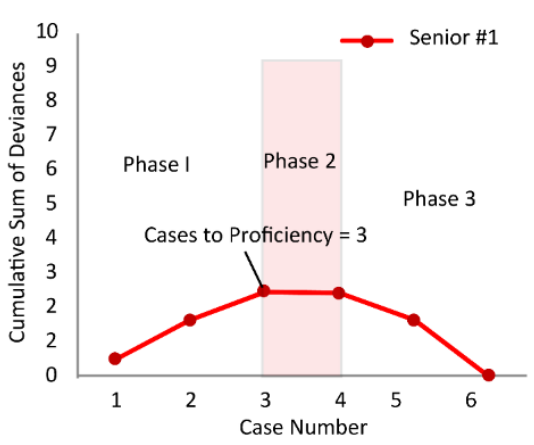

B

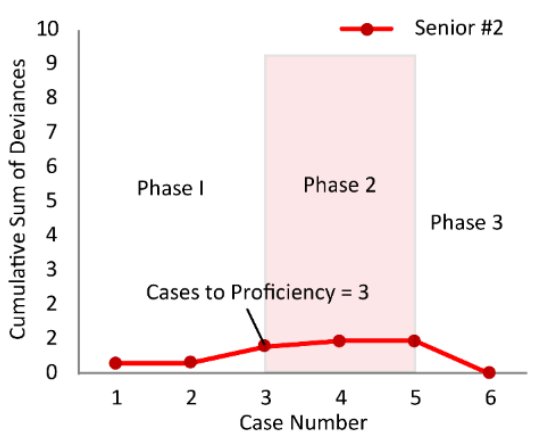

C

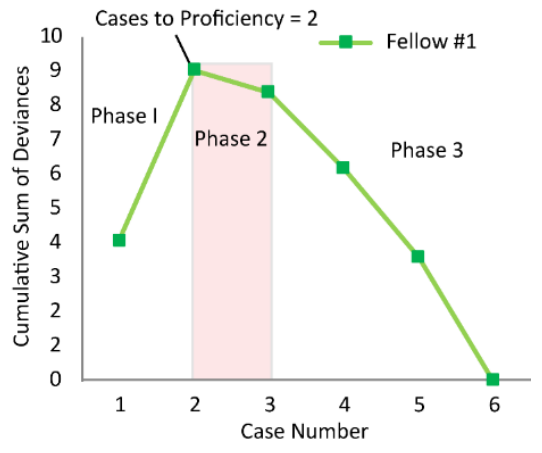

D

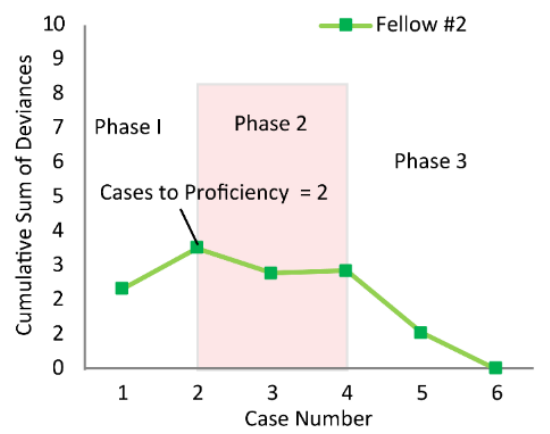

Figure 1. Graphs on the CUSUM deviance charts for A) senior surgeon \#1, B) senior surgeon \#2, C) fellow surgeon \#1, and D) fellow surgeon \#2. The fellow surgeons exhibited a steeper learning curve compared to the senior surgeons. The graph was plotted according to the chronological case numbers. 


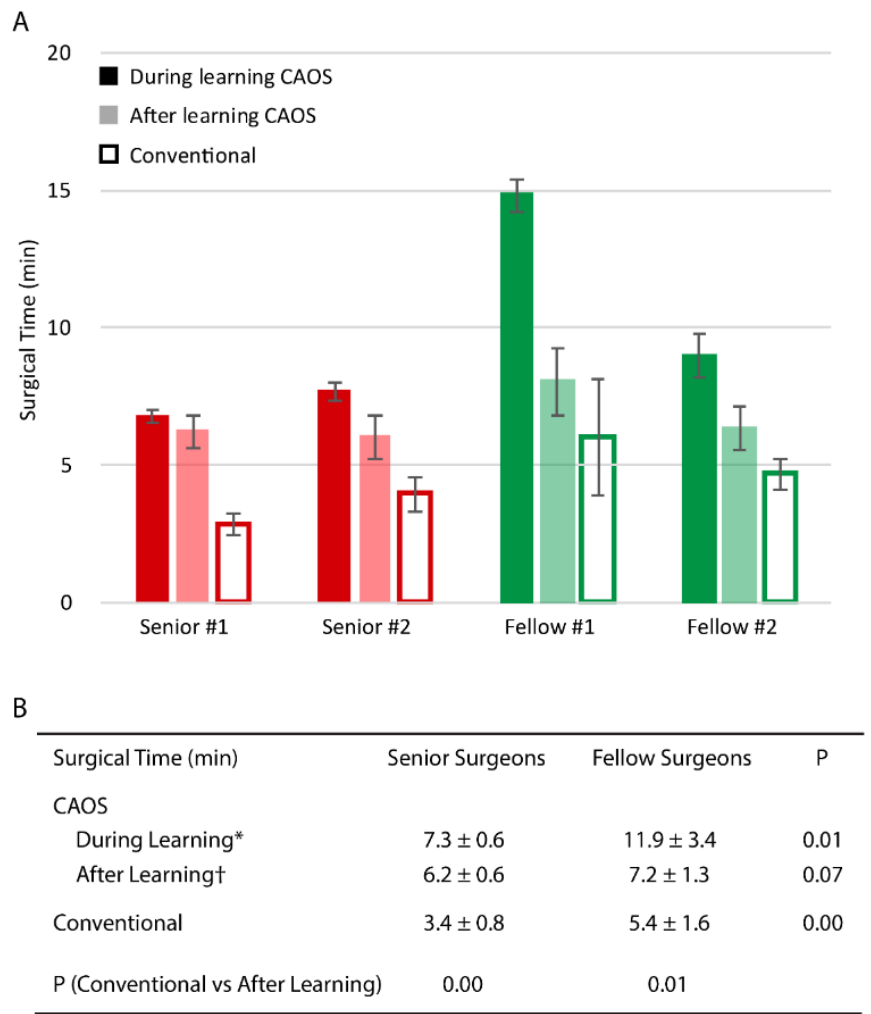

* Calculated as the average of all learning cases (combining all surgeons' cases \#1 - \#CP). + Calculated as the average of all after-learning cases (combining all surgeons' cases \#CP+1 - \#6).

Figure 2. A) Comparison of surgical time between conventional, during learning, and after learning case groups in each individual surgeon. B) Summary of learning characteristics in the senior and fellow surgeon groups.

\section{References}

[1] Sparmann M, Wolke B, Czupalla H, et al. Positioning of total knee arthroplasty with and without navigation support: a prospective, randomised study. J Bone Joint Surg Br. 2003;85(6):830-5.

[2] Petrera P, Petrera J, Siler X, et al. Exactechgps guidance system does not increase operative time when compared to conventionally instrumented total knee arthroplasty. CAOS 2015

[3] Jenny JY, Miehlke RK, Giurea A. Learning curve in navigated total knee replacement. A multicentre study comparing experienced and beginner centres. Knee. 2008;15(2):80-4.

[4] Bokhari MB, Patel CB, Ramos-Valadez DI, Ragupathi M, Haas EM. Learning curve for roboticassisted laparoscopic colorectal surgery. Surg Endosc. 2011;25(3):855-60.

[5] Chaput de Saintonge DM, Vere DW. Why don't doctors use CUSUMs? Lancet. 1974;1:120-1.

[6] Wohl H. The CUSUM plot: its utility in the analysis of clinical data. N Engl J Med. 1977;296:

1044-5.

[7] De Gori M, Adamczewski B, Jenny JY. Value of the cumulative sum test for the assessment of a learning curve: Application to the introduction of patient-specific instrumentation for total knee arthroplasty in an academic department. Knee. 2017;24(3):615-21. 